\title{
Dense consolidations
}

\author{
Consolidação densa
}

\section{Edson Marchiori ${ }^{1,2}$, Gláucia Zanetti ${ }^{2,3}$, Bruno Hochhegger ${ }^{4,5}$}

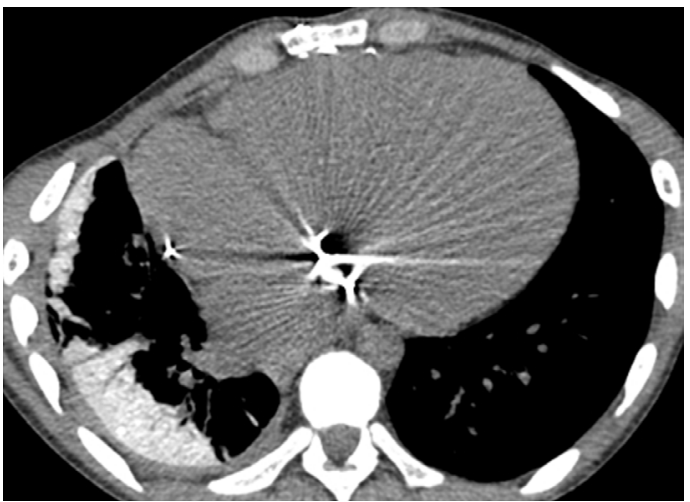

Figure 1 - Axial CT slice of the lung bases, showing increased cardiac volume, small right-sided pleural effusion, and dense consolidation in the lower lobe of the right lung with air bronchogram. The liver parenchyma was also hyperdense (not shown).

A 47-year-old male, previously diagnosed with dilated cardiomyopathy and ventricular tachyarrhythmia, presented with dry cough and progressive dyspnea. Laboratory tests were normal.

A CT of the chest showed a pattern consisting primarily of hyperdense consolidation and cardiomegaly. Consolidation is one of the most common findings on chest CT. Consolidations often have soft parts (with densities similar to those of the heart and liver). This finding is nonspecific, being associated with various diseases. Consolidations can be hypodense (indicative of fat), a finding that is generally characteristic of lipoid pneumonia, or hyperdense (as in the present case). A finding of hyperdense consolidations narrows the differential diagnosis. Hyperdense consolidations are seen in pulmonary alveolar microlithiasis, parenchymal amyloidosis, talc pneumoconiosis, metastatic pulmonary calcification, and amiodarone-induced pulmonary toxicity. The differential diagnosis can be made on the basis of clinical and tomographic characteristics, if those characteristics are evaluated carefully.

A striking feature of alveolar microlithiasis is the dissociation between the clinical and radiological findings, characterized by extensive involvement of the lung parenchyma in patients who are asymptomatic or only mildly symptomatic. The tomographic patterns that facilitate the diagnosis of alveolar microlithiasis include small dense nodules, as well as linear calcification along the interlobular septa and pleural surface. In parenchymal amyloidosis, there is usually a severe clinical profile, accompanied by progressive dyspnea. Nodular thickening of the interlobular septa is highly indicative of a diagnosis of parenchymal amyloidosis. In talc pneumoconiosis, the tomographic findings can be similar to those seen in silicosis (small nodules, conglomerate masses, and subpleural nodules). Talc pneumoconiosis is seen in individuals who work in the talc industry, as well as in injection drug users (after intravenous injection of solutions prepared from tablets containing talc). Metastatic pulmonary calcification, observed in patients with hypercalcemia, is typically secondary to chronic renal failure or secondary hyperparathyroidism. It can also be observed in patients with extensive lytic bone lesions. One CT finding that is quite typical of metastatic pulmonary calcification is that of ill-defined ground-glass, centrilobular nodules with central foci of calcification.

Amiodarone is a drug that is widely used in patients with tachyarrhythmias that are refractory to conventional treatment. The drug contains iodine and tends to accumulate in multiple organs, including the liver and lungs. Amiodarone-induced pulmonary toxicity is one of the serious adverse effects of its use. The tomographic findings that can suggest a diagnosis of amiodarone-induced pulmonary toxicity are cardiomegaly and a hypodense liver. In patients known to have been exposed to amiodarone, tomographic findings of dense consolidations in combination with a dense liver are diagnostic of the condition, precluding the need for a lung biopsy.

\section{Recommended reading}

1. Webb WR, Muller NL, Naidich DP. High-Resolution CT of the Lung. 5th ed. Philadelphia: Wolters Kluwer Health; 2015.

1. Universidade Federal Fluminense, Niterói, Brasil.

2. Universidade Federal do Rio de Janeiro, Rio de Janeiro, Brasil.

3. Faculdade de Medicina de Petrópolis, Petrópolis, Brasil.

4. Santa Casa de Misericórdia de Porto Alegre, Porto Alegre, Brasil.

5. Universidade Federal de Ciências da Saúde de Porto Alegre, Porto Alegre, Brasil. 